\title{
PSICOLOGÍA CLÍNICA Y PSICODIAGNÓSTICO: SOBRE LA VIGENCIA DEL MODELO DE EVALUACIÓN CONDUCTUAL.
}

\author{
Wenceslao Peñate \\ Facultad de Psicología \\ Universidad de La Laguna -Campus de Guajara-
}

\section{RESUMEN}

En el presente artículo se discute la vigencia del modelo de evaluación psicológica, denominado evaluación conductual. Atendiendo a consideraciones conceptuales y prácticas, se discuten las críticas que se han hecho a este modelo. Desde un punto de vista conceptual las críticas se fundamentan en un situacionismo no aplicable a muchos de los problemas psicológicos, la no utilidad del análisis funcional de conducta y la no consideración de las características de personalidad. Desde el punto de vista práctico se discute la utilidad de la técnicas derivadas del modelo conductual, especialmente la observación directa, y la utilidad de relacionar directamente la evaluación con la planificación del tratamiento psicológico. A pesar de esas críticas, se considera que el análisis funcional de conductas es más un proceso de evaluación que una teoría del funcionamiento humano, que las técnicas derivadas del modelo conductual continúan siendo utilizadas (y con frecuencia) y que evaluar cada caso para diseñar un programa de intervención psicológica para cada persona es un recurso que no puede ser desdeñado por la psicología clínica.

Palabras clave: Evaluación Psicológica, Evaluación Conductual, Psicología Clínica. 


\section{ABSTRACT}

In the present article the use of a model of psychological assessment, called behavioral assessment, is discussed. According to both conceptual and practical foundations, the criticism about this model are examined. From a conceptual point of view, the criticism are based on a situationism nonapplicable to many of the psychological problems, the utility of the functional analysis of behavior, and the (supposed) rejection of personality characteristics from this model. From a practical point of view, the utility of the techniques derived from the behavioral model, especially the direct observation, and the utility to relate directly assessment and the planning of the psychological treatment, is questioned. In spite of those critics, it is considered that the functional analysis of behavior is more an assessment procedure than a theory of the human functioning. The assessment techniques derived from the behavioral model continue being used (and frequently,) and that to assess each case to design a psychological intervention program matched to every person is a resource that cannot be scorned by clinical psychology.

\section{Keywords: Psychological Assessment, Behavioral As- sessment, Clinical Psychology}

La evaluación psicológica ha estado vinculada en su devenir histórico al desarrollo de una serie de modelos de evaluación, que se han ido sobreviniendo de una manera dialéctica en una sucesión de respuestas a las insuficiencias del modelo o modelos precedentes. Esta sucesión se ha sustentado en dos polos principales: (i) los progresos en el desarrollo del conocimiento en psicología en cada uno de los momentos; y (ii) en las necesidades de índole práctica, como ha sido la necesidad de responder a los distintos hitos profesionales de la psicología.

En ese sentido, se puede hablar de dos grandes hitos en esa profesionalización: cuando a la psicología se le reconoce la competencia para evaluar las capacidades y características personales de 
los seres humanos y, con el advenimiento de la Psicología Clínica, cuando se le reconoce la competencia para tratar los problemas en salud mental'.

Entrando directamente en el segundo hito, el desarrollo de un currículo formativo en Psicología Clínica con competencias profesionales para tratar los trastornos mentales (y no sólo su evaluación), deriva principalmente del segundo polo señalado anteriormente: la necesidad práctica de contar con nuevos profesionales ante la demanda de ayuda para el tratamiento de los problemas mentales.

Se suele citar como razón principal la demanda asistencial por parte de los participantes en la segunda guerra mundial, víctimas de vivencias traumáticas o altamente estresantes. Esta demanda sobrepasó las capacidad de los dispositivos de salud existentes (atención primaria y psiquiatría). La solución fue la de formar a personas con un cierto conocimiento sobre el ser humano, los profesionales de la psicología, desarrollándose los primeros programas de formación en Psicología Clínica ${ }^{2}$. El estado de la psicología en esos momentos (años cuarenta y cincuenta del siglo pasado) permitió definir al psicólogo clínico con un perfil, hoy día muy conocido, como científico y profesional (Conferencia de Boulder Colorado, USA). Añadiendo a las labores de diagnóstico y tratamiento de los trastornos mentales, la investigación.

Uno de los problemas con que se encontró el nuevo psicólogo clínico, encargado ahora del tratamiento de los trastornos mentales, es que no disponía de un modelo de evaluación que le permitiera planificar ese tratamiento, ya que el modelo de evaluación dominante hasta ese momento (el denominado psicométrico o tradicional), se había elaborado con finalidades puramente diagnósticas, lo que daba una visión estática de la persona evaluada, situada dentro de alguna clasificación psicopatológica. Evidentemente, este modelo

1. Da la impresión de que el tercer hito profesional está todavía por llegar: el reconocimiento de competencias para tratar trastornos de la salud, sea de la índole que sea. La denominada Psicología de la Salud posee un status académico y científico incuestionable hoy día, pero la competencia profesional (el desarrollo oficial de un currículo formativo) está por reconocerse. Habida cuenta que en el estado español la Psicología Clínica tiene una vigencia de hace unos pocos años para acá, no hay razones para ser optimistas.

2. Hay que decir que estos acontecimientos se adscriben básicamente al desarrollo de la psicología profesional en los Estados Unidos de América, coincidiendo con el cambio de eje de la psicología de Europa a América. 
cumplía con la necesidad de cubrir la demanda que hasta ese momento se le hacía a la psicología desde el ámbito aplicado: disponer de instrumentos precisos (empíricamente validados) para elaborar un buen psicodiagnóstico. Pero no permitía elaborar un programa de intervención idiosincrásico, entre otras cosas, porque nada había previsto con respecto al tratamiento psicológico.

Las soluciones provenían del modelo médico: aplicación de tratamientos genéricos, con independencia de quiénes los sufrieran. Si no se disponía de información precisa sobre los determinantes y condicionantes de los problemas psicológicos de cada caso en particular, se actuaba de acuerdo al trastorno (y no al trastornado). Los tratamientos basados en el manual (Manual-based treatments, p.e., Kazdin, 1986; Luborsky y DeRubeis, 1984; Wilson, 1996), asumen implícitamente que los tratamientos para cada trastorno pueden ser eficientes con independencia de la persona que lo sufra ${ }^{3}$. Evidentemente, este planteamiento supone una limitación importante porque no establece una relación directa entre evaluación y tratamiento, no toma en cuenta los procesos particulares en cada persona, el inicio y el curso de cada problema.

En este contexto aparece la evaluación conductual como un modelo de evaluación psicológica que iba a dar respuesta a las necesidades evaluativas de los nuevos psicólogos clínicos: una evaluación directamente vinculada con el tratamiento psicológico. Una evaluación que se desarrolla con la finalidad de elaborar la planificación de un programa de intervención, atendiendo a cómo se plasma en cada persona cada problema, con sus condicionantes y determinantes personales y contextuales. A continuación describiremos brevemente este conocido modelo, para después adentrarnos en la vigencia de sus postulados y sus aplicaciones.

3. A pesar de las limitaciones que supone la aplicación de tratamientos basados en el manual, poseen elementos de carácter práctico que no son nada desdeñables y que se abordarán más adelante. 


\section{CONSIDERACIONES GENERALES SOBRE LA EVALUACIÓN CONDUCTUAL}

A grandes rasgos, las características de la evaluación conductual pueden reflejarse en la tabla 1.

Tabla 1. Características del modelo de evaluación conductual atendiendo a diferentes parámetros.

\begin{tabular}{|c|c|c|c|c|c|}
\hline & SUPUESTOS & OBJETIVO & METODOLOGÍA & $\begin{array}{c}\text { TÉCNICAS } \\
\text { DIAGNÓSTICAS }\end{array}$ & $\begin{array}{c}\text { RELACIÓN } \\
\text { CON TRA- } \\
\text { TAMIENTO }\end{array}$ \\
\cline { 2 - 6 } $\begin{array}{c}\text { MODELO } \\
\text { CONDUC- } \\
\text { TUAL }\end{array}$ & $\begin{array}{c}\text { Causas } \\
\text { ambientales- } \\
\text { aprendizaje/ } \\
\text { Modelo } \\
\text { Mocial-natural/ } \\
\text { La conducta } \\
\text { como muestra }\end{array}$ & $\begin{array}{c}\text { Facilitar la } \\
\text { planificación } \\
\text { del tratamien- psicológico }\end{array}$ & $\begin{array}{c}\text { Ideográfica / Análisis } \\
\text { funcional de conducta }\end{array}$ & $\begin{array}{c}\text { Observación } \\
\text { y registro de } \\
\text { conductas } \\
\text { Registros } \\
\text { fisiológicos }\end{array}$ & Directa \\
\hline
\end{tabular}

El modelo conductual parte de la consideración evaluativa de que la conducta tiene sentido en sí misma, la conducta de un individuo es una muestra de su comportamiento y no un signo, una señal, de la existencia de entidades 'superiores', no hay nada que indique la existencia de instancias intrapsíquicas o entidades psicológicas subyacentes, aunque no se cuestiona que determinadas conductas covarien entre sí. Son las configuraciones estimulares, el ambiente y los procesos de aprendizaje los responsables de la conducta. En este sentido, no caben las comparaciones nomotéticas, sino que un individuo se compara consigo mismo, él es su propio nivel de comparación, defendiendo así una posición ideográfica. El modelo de base está a medio camino entre el naturalista (relaciones causa-efecto) y el social (dinámica de las relaciones entre los distintos elementos, comprensión de la conducta), valorándose tanto los aspectos positivos como los negativos.

El énfasis de este modelo en la conducta observable conllevó la utilización de la observación de conductas como una técnica diagnóstica casi única (al menos en los submodelos más radicales que consideraban conducta sólo a la conducta motora). La consideración posterior del triple sistema de respuestas (la posibilidad de que un 
comportamiento tuviera una expresión cognitiva, motora y fisiológica) hizo que se incluyeran otras técnicas diagnósticas. A pesar de ello se desechan las técnicas proyectivas y los cuestionarios de personalidad, que no tienen un interés en sí mismos, sino que buscan instancias científicamente indemostrables (para este modelo).

Por otro lado, el interés clasificatorio del precedente modelo tradicional psicométrico contrasta con la propuesta del modelo conductual de que el diagnóstico debe tener una relación directa con la intervención, debe hacerse con la finalidad de facilitar la implementación de un programa terapéutico. El análisis funcional de conductas trata de establecer los enlaces funcionales entre el ambiente físicosocial y las conductas objeto de evaluación, con el fin de conocer sus determinantes y condicionantes y, con ello, la facilitación de la planificación de un programa de intervención. La evaluación, que debe continuarse también en la fase de intervención, posibilitará al mismo tiempo una medida de la eficacia de programa implementado, informando a su vez de las posibles modificaciones a llevar a cabo para mejorar o maximizar su eficacia.

Varias décadas después, la vigencia del modelo de evaluación conductual se cuestiona, poniéndose en discusión prácticamente todos sus postulados, así como su aplicabilidad a un buen número de patologías y que ha venido reconociéndose incluso desde los defensores ese modelo (Bellack y Hersen, 1988; Cone, 1993; Goldstein y Hersen, 1998; Haynes, 1998; Pelechano; 1993). Aún cuando la vigencia del modelo se empezó a cuestionar con anterioridad, la escenificación de esa crisis posiblemente se realizará en 1992 con la desaparición de la revista Behavioral Assessment (Cone, 1992).

\section{RAZONES PARA UNA CRISIS}

Como se ha indicado, tratar de identificar el status de la evaluación conductual supone analizar tanto sus componentes conceptuales como los componentes de alcance práctico. Los aspectos críticos de estos dos componentes permiten denominarlos como crisis. 


\section{CRISIS CONCEPTUAL}

Este modelo general de evaluación conductual partía de una serie de fundamentos que fueron cuestionados por su aplicación limitada al análisis psicológico. Algunas de esas limitaciones se pueden observar en los planteamientos situacionistas, el análisis funcional de conducta, la aplicabilidad del triple sistema de respuestas, el papel de las variables de personalidad y las nuevas clasificaciones síndrómicas.

\section{SITUACIONISMO}

Con respecto a los planteamientos situacionistas, desde los modelos conductuales se ha acentuado la determinación por parte del ambiente inmediato de la conducta humana. Estos planteamientos parecen tener sentido en la explicación de algunos problemas conductuales, donde existe un referente externo elicitador del problema (p.e., las fobias). Sin embargo, muchos otros problemas no podían ser explicados atendiendo al contexto inmediato. En ocasiones el historial de aprendizaje, de socialización, explicaban mejor el desempeño actual que la situación que en ese momento esté rodeando al individuo (p.e., las habilidades interpersonales, los estilos de afrontamiento). En ese sentido, el ambiente inmediato debía ser ampliado al ambiente mediato para dar cuenta de los problemas actuales (Fernández-Ballesteros y Staats, 1992). Sin embargo, ahí no se acababan los problemas del situacionismo, porque incluso en los problemas donde mejor se plasmaba ese situacionismo (el caso de la ansiedad fóbica citada) se planteaban dudas sobre la existencia de una predisposición (genética) para las mismas (Merckelbach y de Jong, 1997). El papel de las variables biológicas no estaba resuelto por el modelo conductual, a pesar de que el concepto de predisposición (preparedness, diátesis-estrés), parecía evidente en trastornos psicológicos no vinculados a las psicosis. En cualquier caso, más allá del status de esas variables en el modelo conductual, su presencia en el modelo arranca casi con la generación del mismo, formando parte de la explicación del comportamiento humano (Kanfer y Saslow, 1965). 


\section{ANÁLISIS FUNCIONAL DE CONDUCTAS}

El análisis funcional de conductas (AFC) planteaba un modelo lineal de explicación y predicción de la conducta (antecedentes-conducta-consecuentes) que tampoco era posible verificar en varios de los trastornos psicopatológicos. Sin embargo, en la reconceptualización que hicieron Haynes y O'Brien, (2003), Haynes y O'Brien (1990) y O'Brien y Haynes (1997), se adelantaron ya algunos de los problemas del análisis conductual. Por un lado, como se ha señalado, no todos los problemas evidencian el mismo situacionismo de base. Pero, incluso integrando los conceptos de historial de aprendizaje (ambiente lejano) y de predisposición biológica, todavía quedaban algunos problemas para los que no se encontraba ese disparador inmediato. Tomando como ilustración un trastorno tan frecuente como la depresión, ya hace tiempo que un experto de orientación conductual (Rehm, 1988), indicaba que no era sencillo identificar cuál o cuáles eran los disparadores situacionales que antecedían (explicaban) la ocurrencia de los distintos comportamientos depresivos. De hecho, para este autor, lo característico de la depresión no era su dependencia de una situación determinada, sino del tiempo (las manifestaciones depresivas aparecen y se mantienen en periodos de tiempo concretos). En una revisión posterior sobre depresión melancólica (endógena) y no melancólica (Leventhal y Rehm, 2005), se cuestiona la presencia de estímulos desencadenantes inmediatos, a favor de estresores como precipitantes de las crisis depresivas, con tres particularidades: una, los estresores son más proclives a precipitar crisis depresivas de carácter exógeno; dos, que esos estresores pueden ser más mediatos que inmediatos (abusos físicos y/o sexuales en la infancia, negligencia en la crianza por parte de los padres, conflictos de pareja de los padres, rechazo...); y tres, que no siempre se encuentran esos eventos vitales estresantes en todos los procesos depresivos.

Siguiendo con el modelo lineal, dejando para después las distintas expresiones de la conducta, el mantenimiento de un problema tenía que ver con los refuerzos o consecuencias que seguían a esa conducta. Generalmente se caracterizaban por reforzamiento positivo (conseguir recompensas tangibles, sociales o de bienestar sub- 
jetivo) o por reforzamiento negativo (evitar la presentación de un estímulo punitivo). De nuevo, lo que ocurre es que existían problemas y conductas que no evidenciaban esa dependencia de las consecuencias, al menos de la manera que se expresaba en los modelos conductuales tradicionales. Ejemplos como los del comportamiento psicótico (especialmente en los denominados 'síntomas negativos' de la esquizofrenia), o de algunas compulsiones pueden servir para cuestionar el hecho de que todo comportamiento (incluyendo el psicopatológico) se mantiene por las consecuencias que le siguen. Sin embargo, desde los mismos planteamientos conductuales la teoría del marco relacional (Hayes, Barnes-Holmes y Roche, 2001; Hayes y Ju, 1998), se han actualizado los conceptos skinnerianos de comportamiento gobernado por contingencias y el comportamiento gobernado por reglas. En este último caso, los seres humanos aprendemos una serie de reglas verbales que pueden funcionar como reguladoras de algunos de nuestros comportamientos, incluso frente a la presentación de contingencias inmediatas con un valor reforzante estimable, debido a que esos comportamientos están controlados por la regla verbal. Es probable, en este sentido, que diferentes comportamientos psicopatológicos estén gobernados por reglas, sin que las contingencias inmediatas puedan afectarle (apreciablemente), al tiempo que justificarían muchos de los comportamientos que tratan de aliviar o eliminar el dolor, la tristeza o el sufrimiento psicológico en general (Luciano y Hayes, 2001).

En este punto puede ser interesante retomar la aplicación práctica de la propuesta elaborada por Stephen Haynes sobre modelos causales analiticos funcionales (FACM, Functional Analytic Causal Models). Sobre de la formulación del caso individual por medio de una metodología clínico-analítica funcional (Haynes y O'Brien, 2003; Sturmey, 2008; Virués-Ortega y Haynes, 2005), en la que colocan al AFC como un proceso de evaluación más que como una teoría del comportamiento humano. Como ya se señaló, la búsqueda de antecedentes y consecuentes no se lleva a cabo por la necesidad de su existencia, sino porque, si se pueden localizar posibles enlaces funcionales, éstos facilitarían la introducción de un tratamiento determinado (modificación de la conducta), evitando la confusión entre determinación funcional y causalidad. Entre esos enlaces funciona- 
les incluyen cualquier relación de covarianza. En ese sentido, (tal y como lo señalará Pelechano, 1996), las relaciones de covarianza pueden ser correlacionales o (estrictamente) funcionales y se pueden establecer tanto entre antecedentes-conducta o conducta-consecuentes, como entre conducta-conducta, entre los antecedentes o entre consecuentes y antecedentes, con independencia de la naturaleza de las mismos. Además, se acepta la probabilidad de cambios en el tiempo de estos enlaces. El resultado final es que el modelo puede tener mayor capacidad de explicación y predicción, pero al mismo tiempo está dejando de ser lineal.

\section{TRIPLE SISTEMA DE RESPUESTAS}

Con respecto al triple sistema de respuestas, su aceptación también ha generado dificultades a la hora de adaptarlo a todo comportamiento psicopatológico. El primero de ellos ha sido la constatación de la no covariación entre las tres modalidades de respuesta, cognitiva, motora y fisiológica. Esto significa que la alteración de un sistema no conlleva necesariamente una alteración de magnitud similar en los otros dos. Pero aún más, puede darse incluso que las relaciones fueran negativas o que no existieran, pudiendo un comportamiento expresarse en los tres sistemas, en dos o sólo en una modalidad de respuestas, condiciones éstas que ya fueron detectadas por el modelo de evaluación conductual (Haynes y Wilson, 1979). Esto conlleva un segundo problema: en ocasiones, intentar encontrar la triple expresión de un problema podía significar un esfuerzo innecesario o alterar la propia naturaleza de ese problema.

Un tercer problema planteado por la adopción del triple sistema de respuestas ha sido la función asignada a los componentes no motores, especialmente el componente cognitivo ${ }^{4}$ : las variables cognitivas se han tenido en cuenta como variables procesuales, como aquellas que pueden explicar los distintos cursos de acción de un grupo de personas ante situaciones similares, pero no como conductas-objetivo de la intervención, lo que en la práctica ha supuesto

4. También se puede comentar que el componente fisiológico despertó una serie de esperanzas metodológicas, basadas especialmente en la objetividad de la medida, que posteriormente no se han visto corroboradas ni por su evaluación en los distintos problemas ni por ser una técnica de uso frecuente. 
una jerarquía teórica, basada en una preeminencia del componente motor. El divorcio entre este planteamiento y la realidad psicológica y clínica-psicopatológica parecía evidente: muchas terapias, denominadas cognitivo-conductuales, se han dirigido, a veces casi en exclusiva, al componente cognitivo ${ }^{5}$. Pero de hecho la evaluación conductual no ha desdeñado ese componente oréctico-cognitivo.

En este último sentido se plantea el papel de las variables de personalidad y los síndromes psicopatológicos. La covariación regular de conductas, pensamientos y afectos venía siendo negada por los planteamientos conductistas más extremos, cuando esa covariación era utilizada para derivar la existencia de estructuras latentes o subyacentes (rasgos, dimensiones, estilos o síndromes). Pero no es el caso de que fuera descartada desde la evaluación conductual como objetivo de análisis y, eventualmente, objetivo de intervención.

En este sentido, con el concepto de conductas-clave (keystone behavior, Nelson y Hayes, 1986) o los constructos conductuales (Evans, 1986) se produce un cambio importante. En ambos casos se apoyaba la existencia de covariación entre conductas, que esa covariación tenía cierta nomoteticidad (covarían casi siempre las mismas conductas entre sí) y que podía alterarse una conducta modificando otra con la que covariaba ${ }^{6}$. Algunos autores propusieron que se utilizara esa covariación como un criterio para elegir la conducta objeto de intervención, entre otros posibles criterios. Cone (1986), por ejemplo, sugirió que se tomaran en cuenta criterios estadísticos (y no sólo clínicos o sociales) para elegir esa conducta-clave objetivo de la intervención, de tal forma que las conductas que mantenían magnitudes de correlación con otras fueran elegidas preferentemente. Similarmente, la propuesta de Stephen Haynes sobre el modelo de análisis clínico funcional FACM iba a asumir esos elementos como criterios a tomar en cuenta a la hora de seleccionar el objetivo de la intervención (Haynes y O’Brien, 2003; Heiby y Haynes, 2003;

5. También es cierto que existen aproximaciones terapéuticas que cada vez acentúan más el hacer que las propias cogniciones, incluso en problemas tan cognitivos como la depresión (Martell, Addis y Jacobson, 2001; Wilson y Luciano, 2002).

6. De hecho lan Evans (1986) declara que la aceptación de la covariación era algo asumido por los evaluadores conductuales: "Es obvio que la evaluación conductual utiliza constructos y que ninguna ciencia de la conducta ni ningún modelo de medición conductual podría existir sin hacerlo." (p. 135). 
Virués-Ortega y Haynes, 2005). Lo que quedaba por aceptar era que fuera el conjunto de esa covariación el objetivo de la intervención. En otras palabras, que si se aceptaba que un conjunto de conductas podía covariar, entonces era probable que estuvieran representando un elemento psicológico de orden superior.

La aceptación de esas invariantes más o menos permanentes, llámense estructuras, disposiciones, constructos, procesos, o repertorios comportamentales básicos (Bandura, 1986; Cone, 1986; Fernández-Ballesteros y Staats, 1992; Mischel y Shoda, 1995; Pelechano, 1988) retomó el tema de la evaluación de características psicológicas que eran una función de la persona (y no de la situación). Estas características de personalidad relativamente invariantes van a ser (re)introducidas en los modelos de evaluación como variables mediadoras, moduladoras, o con-causales del desempeño comportamental. Pero, mucho más interesante, en algunos casos incluso pueden ser consideradas como objetivos de la intervención psicológica, proponiéndose desde los propios modelos de personalidad, las estrategias precisas para ello (Adams, Jendritza y Kim, 2005; Matthews, Saklofske, Costa, Deary y Zeidner, 1998; Widiger y Costa, 1994). Lo cierto es que el modelo de evaluación se hace mucho más complejo, no sólo por el añadido que supone el tener que evaluar nuevas variables, sino porque aumenta considerablemente el volumen de interacciones y mediaciones entre los factores situacionales y biológicos sobre los hábitos, los afectos y las cogniciones. El desarrollo de las clasificaciones sindrómicas, ya citadas, colabora desde el campo clínico a la aceptación de esa complejidad evaluativa.

\section{LA CRISIS PRÁCTICA}

Las consideraciones sobre los fundamentos de la evaluación conductual no sólo se han circunscrito, como hemos comentado, a los fundamentos conceptuales, sino también al uso que ha tenido este modelo en el campo aplicado. Supuestamente, si éste era el modelo que unía directamente la evaluación a la planificación del tratamiento, su uso por parte de los psicólogos clínicos-aplicados debería ser mayoritario. De manera general, para verificar este apartado se han analizado dos componentes principales: el uso de de- 
terminados procedimientos y técnicas diagnósticas de acuerdo con el modelo, y el uso de la evaluación para la planificación de un tratamiento psicológico individualizado.

\section{TÉCNICAS Y PROCEDIMIENTOS DE EVALUACIÓN}

Con respecto al uso de una metodología diagnóstica determinada, Fernández-Ballesteros, Zamarrón y Huici (1992) llevaron a cabo un estudio año a año desde 1974 hasta 1991 (referenciado en Fernández-Ballesteros, 1993), sobre las publicaciones dentro del modelo de evaluación conductual. Del análisis de este trabajo se puede extraer la conclusión de que se está publicando más en el último periodo (140 artículos en 1991) que en el primero (25 en 1980) y, por tanto, no se puede, en principio, hablar de crisis. Pero si se toma todo el continuo, lo que se observa es una asíntota en 1983 (210 artículos) seguida de 1984 (175) y un cierto decline hasta llegar a 1991. En este caso se puede decir que, a partir de 1984 se produce una disminución de los estudios sobre evaluación conductual, fenómeno que podría ser un reflejo de la crisis de esta disciplina.

Sin embargo, en 1992 y 1993, John Cone, a propósito de la desaparición como publicación independiente de la revista Behavioral Assessment y de la crisis de la evaluación conductual, analiza los contenidos de los artículos científicos durante distintos periodos. En concreto, Cone (1993) examina en dos periodos de tiempo (198082 y 1990-92), los contenidos sobre métodos de evaluación de los artículos recogidos en el PsychoLIT. En la tabla 2 presentamos los datos, extraídos de su informe. 
Tabla 2. Resumen del estudio de John Cone sobre el uso de determinados procedimientos y técnicas diagnósticas en artículos científicos, publicados en dos periodos de tiempo.

\begin{tabular}{|c|c|c|c|c|}
\cline { 2 - 5 } \multicolumn{1}{c|}{} & \multicolumn{4}{c|}{ PERIODO } \\
\cline { 2 - 5 } & \multicolumn{2}{|c|}{$1980-82$} & \multicolumn{2}{c|}{$1990-92$} \\
\hline MÉTODO & $N^{0}$ artículos & $\%$ & $N^{\circ}$ artículos & $\%$ \\
\hline Entrevista & 7 & 5,8 & 10 & 7,2 \\
\hline Autoinformes & 11 & 9,2 & 19 & 13,7 \\
\hline Calificación por otros & 31 & 25,8 & 47 & 33,8 \\
\hline Autorregistro & 7 & 5,8 & 9 & 6,5 \\
\hline Observación directa & 64 & 53,3 & 54 & 38,8 \\
\hline Total & 120 & 99,9 & 139 & 100 \\
\hline
\end{tabular}

Modificado de Cone (1993).

Con estos datos, el autor considera que, en primer lugar, no sólo no han disminuido las publicaciones en evaluación conductual, sino que su orientación instrumental se ha mantenido en los mismos términos, representando el principal objeto de estudio la observación directa (el estudio de la observación es más apreciable si se suma el autorregistro a la observación directa). En segundo lugar, se podría decir que la crisis procedimental es más aparente que real: ni ha disminuido el interés por el estudio de la metodología, ni la observación sistemática ha sido paulatinamente abandonada.

Esa podía ser la situación desde el punto de vista de las publicaciones científicas, pero, ¿Qué estaba ocurriendo en el mundo aplicado? Hace tiempo realizamos una encuesta sobre una muestra de los profesionales de la Psicología que ejercían en S/C de Tenerife (Peñate y González, 1995). Preguntados sobre qué técnicas y procedimientos diagnósticos utilizaban, las respuestas de los 90 encuestados fueron las siguientes: 
Tabla 3. Porcentaje de profesionales que utilizan determinados procedimientos o técnicas de evaluación. $(N=90)$.

\begin{tabular}{|lc|}
\hline Entrevista & $95,6 \%$ \\
Tests de Personalidad & $90,0 \%$ \\
Autorregistro & $57,8 \%$ \\
Observación directa $^{\text {Autoinformes conductuales }}$ & $51,1 \%$ \\
Entrevista con allegados $^{1}$ & $46,7 \%$ \\
Juego de Roles $^{\text {Tests Proyectivos }}$ & $41,1 \%$ \\
\hline
\end{tabular}

Como puede observarse, las técnicas que se podrían denominar 'más conductuales' (observación, autorregistro, autoinformes conductuales, juego de roles) mantienen un uso relativamente frecuente. De todas formas, lo que destaca para nosotros es que, consolidado el modelo conductual, sorprendentemente, los tests psicométricos son utilizados por el $90 \%$ de los que respondieron a la encuesta, siendo el segundo procedimiento diagnóstico más utilizado (después de la entrevista) y a mucha distancia de los denominados 'conductuales'.

Ante estos resultados se podría concluir que el modelo conductual no ha logrado implantarse completamente dentro de la práctica profesional, por lo menos en lo que hace referencia a la instrumentación diagnóstica utilizada y todo ello a pesar del tiempo transcurrido desde su formulación. Ciñéndonos a nuestro trabajo, destaca el uso de la triada entrevista, tests psicológicos y autorregistro. Los autoinformes conductuales se utilizan la mitad que los tests psicológicos. Otro dato que podría ser interesante sería conocer cómo ha ido evolucionando el uso de la instrumentación diagnóstica. En ese sentido, en la misma encuesta anterior se preguntó a los 90 profesionales sobre el abandono de técnicas o procedimientos de evaluación utilizados con anterioridad (en el caso de que respondieran que habían utilizado alguna técnica alguna vez, se les preguntaba si la seguían utilizando en la actualidad). Los datos obtenidos son los que se presentan a continuación. 
Tabla 4. Porcentajes de profesionales han abandonado el uso de determinada técnica o procedimiento $(\mathrm{N}=90)$.

\begin{tabular}{|l|c|}
\hline TÉCNICA O INSTRUMENTO & Abandonos \\
\hline Entrevista & 3,5 \\
\hline Tests de personalidad & 7,1 \\
\hline Autorregistro & 11,1 \\
\hline Autoinformes conductuales & 13,0 \\
\hline Tests proyectivos & 14,9 \\
\hline Observación directa & 19,2 \\
\hline Escalas de calificacion por otros & 20,9 \\
\hline Juego de roles & 23,3 \\
\hline Registros psicofisiologicos & 36,8 \\
\hline
\end{tabular}

Abandonos $=\%$ de profesionales que han dejado de utilizar esa técnica o instrumento

Como puede observarse, la evolución no ha ido precisamente en el sentido de un incremento de las técnicas más conductuales. Los procedimientos que han tenido menos abandonos han sido los señalados anteriormente como de mayor uso: la entrevista, los tests de personalidad y el autorregistro, seguidos de los autoinformes conductuales. Procedimientos como la observación, el juego de roles o los registros psicofisiológicos son los que mayor 'deserción' provocan. Parecería por tanto que más que una paulatina aceptación de las recomendaciones del modelo conductual, lo que se vislumbra es un cierto descrédito de alguna de sus recomendaciones procedimentales (especialmente la observación sistemática en condiciones naturales).

Sin embargo, se podría indicar que, a pesar de que la gran mayoría de los profesionales se declaran de orientación cognitivo-conductual, no todos lo son y no todos serían igual de rigurosos, lo que justificaría ese tipo de uso de la metodología diagnóstica vinculada al modelo conductual. Si tomáramos en cuenta entonces la mejor práctica posible los datos podrían cambiarse. Un modo de conocer esa mejor práctica posible podría derivarse de los estudios de casos publicados 
en revistas científicas. En nuestro caso hemos hecho un registro de los instrumentos, procedimientos y técnicas diagnósticas utilizadas en los casos publicados en la revista de Análisis y Modificación de Conducta (es de las escasas en psicología que tiene un apartado en este sentido), tomando como referencia la década posterior al estudio de John Cone: 1992-2002. Los datos en porcentajes de uso de algunos de esos procedimientos se resumen en la figura 1.

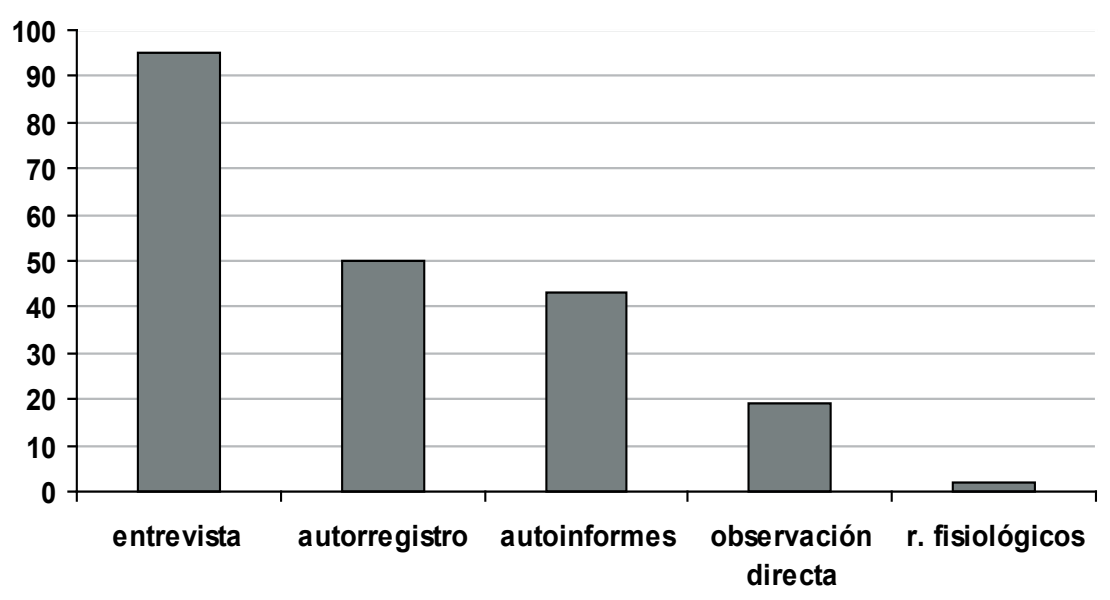

Figura 1. Porcentaje de uso de determinados procedimientos evaluativos en los casos publicados por la revista Análisis y Modificación de Conducta, comprendidos en el periodo de 1992-2002.

Como puede observarse, si dejamos al margen la entrevista (sólo en contados casos no se utilizó por las características de la persona evaluada), la auto-observación y la observación directa supone casi un $70 \%$ de uso, En el caso del autorregistro, su uso es superior a los autoinformes. Lo que sí parece que ha caído en desuso son los registros psicofisiológicos.

Acudiendo a esa diferenciación tradicional entre el científico y el profesional, se podría argumentar que lo que pasa es que el mundo profesional es menos riguroso y menos preciso que el mundo científico y que, ante las dificultades y costos temporales de las técnicas conductuales, los profesionales optan por soluciones menos riguro- 
sas, pero más rápidas y sencillas (eso explicaría el abandono de la observación en condiciones naturales y los registros psicofisiológi$\cos y$, en cambio, el uso de los autoinformes conductuales y autorregistro). Otras consideraciones, referidas a la no estandarización de los procedimientos de observación, sus dificultades procedimentales o su costo de aplicación (en tiempo) se podrían utilizar para justificar la falta de predominio de la observación directa, pero también parece evidente que, si el procedimiento observacional hubiera aportado un avance sustantivo y significativo (frente a otros procedimientos de evaluación), se habría implementado de cualquier forma. Lo que se constata en todo caso, es que las técnicas y procedimientos derivados de la propuesta de la evaluación conductual, continúan vigentes en la práctica profesional.

\section{LA EVALUACIÓN EN RELACIÓN DIRECTA CON EL TRATAMIENTO}

Como se ha venido señalando, una de las aportaciones del modelo conductual al campo de la psicología clínica (y aplicada en general) fue la consideración de que la evaluación conductual unía directamente la evaluación y el tratamiento psicológico. Frente al modelo psicométrico tradicional, que tenía intereses meramente clasificatorios, el modelo conductual iba más allá y no sólo evaluaba para intervenir, sino que de la evaluación se derivaba la elaboración del programa de intervención. Basándose originalmente en el modelo lineal del AFC, se identificaban las condiciones (funcionales) que mantenían una conducta determinada y, en función de la bondad de esa identificación, se podría alterar dicha conducta alterando los elementos que la mantenían.

El hecho de que esta propuesta apareciera en el momento en el que el campo profesional estuviera reclamando un psicólogo clínico no solo evaluador, sino con conocimientos para intervenir en los problemas de salud mental (en las décadas posteriores a la segunda guerra mundial, como ya se mencionó), facilitó su aceptación, en la medida en que este nuevo modelo, que establecía una relación de continuidad evaluación-tratamiento, respondía a esas nuevas demandas profesionales. 
Sin duda, el interés mostrado por la evaluación conductual en relacionar directamente la evaluación y la intervención tenía valor en sí mismo. Estaba claro que un diagnóstico meramente clasificador no sólo no respondía a las nuevas demandas, sino que podría estar funcionando como la antítesis de lo que se promovía ahora (el cambio), ya que el uso inadecuado de las clasificaciones psicopatológicas podrían estar actuando como etiquetas inamovibles e inmodificables. Sin embargo, es posible que también hayamos caído en un cierto maniqueísmo, ya que hoy día no está claro que el diagnóstico clasificatorio no facilite la elaboración de un programa de intervención y tampoco está claro que el modelo conductual se haya utilizado de acuerdo con los postulados que relacionaban directamente la evaluación y la intervención.

Entrando a comentar la relación entre el diagnóstico tradicional y el tratamiento psicológico, ya se comentó cómo algunos expertos (a partir de la elaboración del DSM-III-R), observaban en las nuevas clasificaciones psiquiátricas elementos que favorecían la elaboración de un programa de intervención (First, Frances, Widiger, Pincus y Davis, 1992; Hersen, 1988). Sin embargo, el apoyo mayor a esa relación ha procedido de lo que se ha denominado tratamientos basados en el manual (Wilson, 1996, 1997). De acuerdo con este método, se trata de aplicar unos procedimientos terapéuticos descritos y secuencializados en un manual, a problemas concretos, que responden a una nomenclatura de clasificación psicopatológica (son manuales referidos a problemas específicos como agorafobia/ pánico, depresión, problemas de la conducta alimentaria, trastornos obsesivo-compulsivos, etc., y que han tenido una gran divulgación).

Este planteamiento estaría alejado del AFC, porque no se basa en el análisis del caso individual y de las condiciones contextuales del comportamiento, sino que se basa en la identificación de un problema por medio de una etiqueta diagnóstica y, una vez conocido el trastorno, se aplica el procedimiento genérico que facilita el manual. Es decir, se trataría de situar a la persona dentro de una clasificación psicopatológica y aplicarle un programa que ha podido verificar ${ }^{7}$ su

7. No todos los manuales promueven técnicas de intervención validadas científicamente. Los procedimientos basados en el manual hacen referencia a aquéllos que sí se han verificado experimentalmente. 
utilidad en el control de ese trastorno (pero no en esa persona en concreto). En ambos casos se utiliza un criterio nomotético, alejado de los planteamientos idiográficos de los modelos conductuales.

Aparentemente la utilidad de estos procedimientos debería ser peor que los basados en la formulación del caso individual del modelo de evaluación conductual funcional. Los datos aportados por algunos trabajos y revisiones (cfr., Barlow, 1994; Wilson 1996), evidencian que los resultados de estas intervenciones son satisfactorias, no sólo en el terreno experimental, sino en el clínico aplicado. Quizás en este campo sea donde más destaquen los trabajos de Dietmar Schulte (Schulte, 1996; Schulte, Künzel, Pepping y Schulte-Bahrenberg, 1992). Este autor puso en evidencia cómo la intervención sobre grupos de fóbicos relativamente amplios por procedimientos basados en el manual y por procedimientos basados en la formulación del caso individual, no sólo no mostraban la mejor adecuación de la formulación del caso individual, sino la mayor eficacia de los procedimientos de intervención estandarizados (exposición en vivo con autoinstrucciones), con un $100 \%$ de los casos (sumados los que mejoraron y los que se 'curaron').

Pero lo más llamativo y demoledor para el AFC fue que la formulación del caso individual no fue mejor que un procedimiento aleatorio: los análisis de cada caso en particular del grupo de análisis individualizado, se aplicaron a otras personas con diferentes fobias de manera aleatoria. Tanto en las medidas post-intervención como en el seguimiento, este grupo de análisis individual aleatorio no obtuvo peores resultados que el de análisis funcional individual. Además, estos resultados fueron independientes de la experiencia del terapeuta y del tipo de fobia. Parecería que el mejor papel del terapeuta era de motivar al paciente para el cambio y seleccionar y aplicar el protocolo adecuado.

Los trabajos de D. Schulte no han podido ser corroborados, en sentido estricto, por otras investigaciones sobre otros problemas, aunque sí se ha constatado la eficacia de los tratamientos basados en el manual. Sin embargo, otro grupo de trabajos han mostrado la superioridad de los tratamientos basados en el análisis del caso individual (Ghaderi, 2006; Iwata, Pace, Dorsey, Zarcone, Vollmer, y Smith, 1994). De hecho el propio Schulte ha reconocido que hay ocasiones en las que el manual falla (Schulte y Eifert, 2002). 
La situación por tanto estaría en que la supuesta relación directa entre evaluación y la planificación del tratamiento psicológico, la propuesta de la evaluación conductual en relación con los nuevos papeles de la psicología clínica, no supone un mejor éxito terapéutico que los tratamientos basados en el manual. Sin embargo, un análisis más pormenorizado de estos manuales puede cuestionar esa independencia entre el protocolo terapéutico y el diagnóstico individual previo.

Siguiendo a Wilson (1996, 1997, 2005): (i) los tratamientos basados en el manual no están realmente reñidos con los fundamentos del modelo conductual. De hecho la mayoría de estos manuales son de origen cognitivo-conductual. Estos manuales están basados en los tratamientos empíricamente validados o basados en la evidencia, tal y como ha revelado el trabajo de la Task Force para la American Psychology Association (Chambless et al., 1996; 1998).

(ii) El tratamiento basado en el manual no implica que se utilice el mismo tratamiento (o paquete de tratamientos) para una misma categoría diagnóstica. Con frecuencia el terapeuta tiene distintos itinerarios de actuación de acuerdo con la naturaleza del problema, el curso y la comorbilidad del mismo ${ }^{8}$ y, por qué no, las características de cada persona con sus condicionantes y determinantes. Lo que no está establecido en estos manuales es que se alteren esos distintos cursos de acción de acuerdo con 'la experiencia práctica' de cada terapeuta aún cuando es posible que eso sea lo que ocurre en la práctica (Wilson, 1997). De hecho, hace ya tiempo que se han propuesto soluciones para unificar el análisis del caso individual con los tratamientos basados en el manual (Eifert, 1996; Eifert y Feldner, 2003; Evans, 1996).

(iii) La formación del terapeuta (y el establecimiento de unas adecuadas relaciones terapéuticas) es crucial en la aplicación de los tratamientos basados en el manual. Cualquier manual (p.e., uno de los más conocidos y eficientes como el de Barlow y Craske, 2000, para el pánico y la agorafobia), acentúan el papel del terapeuta en la motivación para el cambio, la adaptación de los protocolos dentro de los distintos cursos de acción y la eventual consolidación de los resultados obtenidos. No parece posible que se pueda establecer una buena alianza

8. Esto es una forma rápida de decirlo, porque los manuales suelen incluir más de un procedimiento terapéutico y reglas para su aplicación secuencializada. 
terapéutica sin que el terapeuta trate de profundizar en el conocimiento de cada caso individual. Lo que puede estar ocurriendo también es que no se han resuelto las preferencias de los pacientes y clientes por determinadas características terapéuticas (edad, sexo, etnia, estado civil...) a la hora de permanecer en un proceso terapéutico, más allá de otros elementos primordiales en la comunicación paciente-terapeuta como el tipo de trastorno, síntomas específicos, desarrollo de la sintomatología (Hassan, McCabe y Priebe, 2007). Esta cuestión puede que no sea independiente de la formación que los profesionales estén recibiendo Como han puesto en evidencia Weissman et al., (2006), los programas de formación de terapeutas no incorporan de manera sistemática la formación en los tratamientos empíricamente validados, compitiendo éstos con otros tratamientos que no se derivan de la investigación o la evidencia clínica9 .

En definitiva, la intervención basada en el manual no es tan indirecta respecto a la evaluación de un problema, la aplicación de los tratamientos basados en el manual no están 'libres' del análisis funcional conductual. Los manuales actuales tienen en su mayoría un proceso de evaluación previo que delimita el modo y el tipo (si los hay disponibles) de la intervención a seguir, que suelen guardar elementos funcionales (condicionantes y determinantes) comunes. En todo caso, si bien es cierto que los tratamientos basados en el manual proporcionan una estrategia diferenciada del análisis de cada caso en particular, propia de la evaluación conductual, no hay razones para pensar que sea más eficaz que el análisis individual.

Lo dicho para los tratamientos basados en el manual no evita que la intervención basada en la formulación del caso individual haya terminado por significar la solución que aporte una mejora significativa en la eficiencia terapéutica. De hecho, quizás esto último sea la clave para entender la crisis de la relación directa entre evaluación e intervención: los niveles de eficiencia terapéutica de los tratamientos disponibles tienen tales limitaciones hoy día (y ese es el estado actual del desarrollo del conocimiento), que podrían justificar que no se observen distinciones entre los basados en procedimientos estandarizados o en el análisis funcional.

9. Este estudio hace referencia a una muestra de programas de formación en USA. No parece que existan muchas razones para pensar que no ocurre lo mismo en el estado español. 


\section{CONSIDERACIONES FINALES}

El resultado del análisis de los componentes internos del modelo de evaluación conductual ofrece un panorama de la situación de crisis del modelo que alcanza a todos sus elementos principales. Sin embargo, no todos esos aspectos críticos parecen de aplicación a la propia evaluación conductual. En la tabla 5 tratamos de resumir ese estado de la cuestión.

Tabla 5. Elementos críticos que cuestionan la vigencia de la evaluación conductual como estrategia diagnóstica para la Psicología Clínica.

\begin{tabular}{|c|l|l|}
\hline ELEMENTO & \multicolumn{1}{|c|}{ VIGENCIA } & \multicolumn{1}{|c|}{ CONSIDERACIONES } \\
\hline Situacionismo & $\begin{array}{l}\text { No todos los problemas psicoló- } \\
\text { gicos muestran una dependencia } \\
\text { del contexto inmediato. }\end{array}$ & $\begin{array}{l}\text { El reconocimiento del papel del } \\
\text { ambiente mediato (aprendizaje, } \\
\text { historial de socialización) ha es- } \\
\text { tado presente en el modelo con- } \\
\text { ductual. }\end{array}$ \\
\hline $\begin{array}{c}\text { Análisis } \\
\text { funcional de } \\
\text { conductas }\end{array}$ & $\begin{array}{l}\text { No todos los problemas eviden- } \\
\text { cian una dependencia lineal an- } \\
\text { tecedentes-conducta-consecuen- } \\
\text { tes. }\end{array}$ & $\begin{array}{l}\text { La búsqueda de los condicionan- } \\
\text { tes y determinantes de la conduc- } \\
\text { ta, antecedentes y consecuentes } \\
\text { es un propósito evaluativo }\end{array}$ \\
\hline $\begin{array}{c}\text { Triple sistema } \\
\text { de respuesta }\end{array}$ & $\begin{array}{l}\text { No existe una prevalencia del } \\
\text { componente motor en todos los } \\
\text { trastornos mentales. }\end{array}$ & $\begin{array}{l}\text { La no sincronía entre los tres ca- } \\
\text { nales de respuestas, así como la } \\
\text { covariación entre distintos tipos } \\
\text { de respuestas ha estado presen- } \\
\text { te dentro del modelo conductual }\end{array}$ \\
\hline $\begin{array}{c}\text { Técnicas y } \\
\text { procedimientos } \\
\text { diagnósticos }\end{array}$ & $\begin{array}{l}\text { Las técnicas y procedimientos } \\
\text { conductuales han perdido vigen- } \\
\text { cia (incluso frente a las provenien- } \\
\text { tes del modelo psicométrico. }\end{array}$ & $\begin{array}{l}\text { Parece depender de la orienta- } \\
\text { ción, necesidades, oportunidad y } \\
\text { formación de los psicólogos clíni- } \\
\text { cos y no de su vigencia. }\end{array}$ \\
\hline $\begin{array}{c}\text { Relación con } \\
\text { el tratamiento }\end{array}$ & $\begin{array}{l}\text { Una relación directa entre evalua- } \\
\text { ción y tratamiento no ha mostrado } \\
\text { ser mejor que los tratamientos ba- } \\
\text { sados en el manual }\end{array}$ & $\begin{array}{l}\text { Los tratamientos individualizados } \\
\text { han mostrado su eficiencia y los } \\
\text { tratamientos basados en el ma- } \\
\text { nual no están libres de ese trato } \\
\text { individualizado. }\end{array}$ \\
\hline
\end{tabular}

Desde el punto de vista conceptual, el modelo de evaluación conductual se ha asentado con frecuencia en planteamientos situa- 
cionistas, pero siempre ha contemplado el papel del ambiente remoto (socialización, aprendizaje anterior). Lo que también puede ser cierto es que este ambiente mediato se rige por las mismas reglas conductuales (no se apela a instancias intrapsíquicas o subyacentes), contemplando el papel mediador del componente biológico, determinante en algunos casos (es impensable que el modelo conductual no considerara el componente biológico en los trastornos que evidencian un deterioro orgánico o neurológico).

El carácter líneal del AFC debe ser entendido como una estrategia de análisis. La búsqueda de antecedentes, preferentemente inmediatos, que elicitan el comportamiento anómalo, y la búsqueda de las consecuencias tangibles, es un propósito evaluativo en la medida en que su localización facilitaría en gran medida el diseño de un programa de tratamiento. Pero es evidente que no todos los problemas evidencian un situacionismo de base y no todos los comportamientos están regulados por contingencias. Aún más, desde los modelos conductuales, existe un volumen apreciable de conocimiento científico de la interdependencia entre Estímulos-Conductas-Consecuencias, con relaciones de determinación recíproca. De nuevo, la linealidad del AFC tiene una intencionalidad, un deseo, de facilitar el conocimiento de un problema (de sus condicionantes y determinantes), no es una declaración de principios, entre otras cosas, porque estaría negando el legado científico del modelo conductual.

De la misma manera, las variables cognitivas y la covariación entre distintos tipos de respuestas se recogen dentro de los modelos de evaluación conductual. Si bien es cierto que se han obviado denominaciones tradicionales para esa covariación (prefiriéndose denominaciones como repertorios, clases, constructos conductuales...). Sin embargo, la integración de las dimensiones de personalidad no están reflejadas en todas las variaciones de los modelos conductuales $y$, salvo excepciones, no se contemplan como objetivos de la intervención o como procesos a la base de un trastorno determinado, sino como variables moduladoras, sin un peso importante en la valoración del potencial de cambio de una persona. Hay que volver a señalar que el énfasis de los modelos conductuales en los elementos observables es una representación de afán por objetivar al máximo la ciencia psicológica, evitando conjeturas y apelaciones a instancias no demostradas. 
Desde el ámbito de la instrumentación de evaluación propuesta, el panorama para el modelo conductual es evidente: la técnica de evaluación "princeps" y más promocionada, la observación directa, no sólo no ha logrado implantarse como la técnica dominante, sino que los propios terapeutas de conducta prefieren otras técnicas o procedimientos (incluyendo los denostados tests psicológicos). Pero este hecho no ha significado que la observación directa haya caído en el descrédito. Parece que, por razones operativas y de optimización de recursos, se ha preferido obtener la información por medio de otros procedimientos (entrevista, autorregistros, autoinformes). Por otro lado, no todo profesional de la psicología clínica posee una orientación conductual, no todos tienen la misma pericia y no todos los actos diagnósticos se hacen con una finalidad de intervención psicológica (de hecho el diagnóstico clasificatorio sigue siendo un área de trabajo productiva para los profesionales de la psicología, donde los tests estandarizados tradicionales juegan un papel muy importante).

Un elemento central del modelo de evaluación conductual era el que establecía una relación directa entre evaluación e intervención. De hecho se vino a sostener en un principio que la única funcionalidad de la evaluación era la de aportar información en la planificación de la intervención. La elaboración de procedimientos reglados de intervención, (manuales prácticos sobre el manejo de distintos trastornos o problemas), y su uso por los terapeutas de conducta también ha supuesto un cambio con respecto al modelo, que alcanza a las bases metódicas del mismo: el análisis ipsativo, idiográfico, la formulación del caso individual se ha cuestionado en la medida en que los terapeutas aplican recetas o manuales sin prácticamente tener en cuenta cada caso individual. Y con una eficacia terapéutica comparable a la formulación del caso individual. Pero el hecho de que sea comparable no significa que la formulación del caso individual no siga teniendo vigencia. Y, aún más: desde los tratamientos basados en el manual se incluye parte de ese análisis individualizado en la toma de decisiones del clínico, al tiempo que juega un papel importante en el establecimiento de una alianza terapéutica más sólida. En estos momentos del conocimiento, donde las características del terapeuta y su estilo de comunicación desempeñan un papel tan importante en 
la relación terapeuta-paciente, parece impensable que, sea cual sea la orientación, no se tome en cuenta ese análisis individualizado.

Con todo y a modo de resumen, no parecen existir razones de peso para cuestionar la vigencia de la evaluación conductual. Evidentemente, con el paso del tiempo y el desarrollo del conocimiento, el modelo de base se ha ido modificando y complicando, pero no ha perdido sus señas de identidad y no ha dejado de ser una estrategia de evaluación psicológica útil: el valor de cada individuo en sí mismo, el empeño en determinar de la manera más objetiva posible los condicionantes y determinantes del comportamiento humano (con independencia de que sean elementos causativos o no) y las relaciones funcionales de esos elementos con las conductas-problema para planificar un tratamiento psicológico individualizado, son propuestas difícilmente rechazables desde una psicología clínica preocupada por el mayor bienestar personal y social de la personas que toma a cargo. En todo caso, ahí sigue estando disponible (Mash y Hamsley, 2003).

\section{REFERENCIAS}

Adams, W., Jendritza, T. \& Kim, S. (2005). Bordeline Personality Disorder. En M. Hersen (Ed.). Clinician's Handbook of Adult Behavioral Assessment (pp. 431-460). Burlington, MA: Academic Press.

Bandura, A. (1986). Social foundations of thought and action: a social cognitive theory. NJ: Prentice Hall.

Barlow, D. \& Craske, M.G. (2000). Mastery of your anxiety and panic ( $3^{a}$ ed.). San Antonio, TX: The Psychological Corporation.

Barlow. D. (1994). Psychological intervention in the era of managed competition. Clinical Psychology, 1, 109-122.

Bellack, A. \& Hersen, M. (1988). Future directions of Behavioral Assessment. En A. Bellack, \& M. Hersen (Eds.). Behavioral Assessment. A practical handbook. New York: Pergamon Press. ( $3^{\mathrm{a}}$ ed.).

Cone, J. (1986). Ideographic, nomothetic, and related perspectives in behavioral assessment. En R. Nelson \& S. Hayes (Eds.). Conceptual foundations of behavioral assessment. New York: Guilford Press. 
Cone, J. (1986). Ideographic, nomothetic, and related perspectives in behavioral assessment. En R. Nelson \& S. Hayes (Eds.). Conceptual foundations of behavioral assessment. Nueva York: Guilford Press.

Cone, J. (1992). That was then! This is now!. Behavioral Assessment, 14, 219-228.

Cone, J. (1993). The current state of Behavioral Assessment. European Journal of Psychological Assessment, 9,175-181.

Chambless, D.L., Baker, M. J., Baucom, D. H., Calhoun, K. S., CritsChristoph, P., Daiuto, A., et al. (1998). Update on empirically validated therapies: II. The Clinical Psychologist, 51, 3-16.

Chambless, D.L., Sanderson, W. C., Shoham, V., Johnson, B., Pope, K. S., Crits-Christoph, P., et al. (1996). An update on empirically validated therapies. The Clinical Psychologist, 49, 5-18.

Eifert, G.H. (1996). More theory-driven and less diagnosis-based behavior therapy. Journal of Behavior Therapy and Experimental Psychiatry, 27, 75-86.

Eifert, G. y Feldner, M. (2003). Conceptual foundation of behavioral assessment strategies. En E. Heiby \& S. Haynes (Eds.). -Comprehensive handbook of psychological assessment: Vol. 3. Behavioral assessment. (pp. 94-107). Nueva York: Wiley.

Evans, I. (1986). Behavioral view of personality. En R. Nelson \& S. Hayes (Eds.). Conceptual foundations of behavioral assessment. New York: Guilford Press.

Evans, I. (1996). Individualizing therapy, customizing Clinical science. Journal of Behavior Therapy and Experimental Psychiatry, 27, 99-105.

Fernández-Ballesteros, R. (1993). Behavioral Assessment: Dying, vanishing or still running? European Journal of Psychological Assessment, 9, 159-174.

Fernández-Ballesteros, R. y Staats, A. (1992). Paradigmatic Behavioral Assessment, Treatment and Evaluation; Answering the crisis of behavioral assessment. Advances in Research Behavior Therapy, 14, 1-28.

First, M., Frances, A., Widiger, T., Pincus, H. \& Davis, W. (1992). DSM-IV and Behavioral Assessment. Behavioral Assessment, 14, 297-306. 
Ghaderi, A. (2006). Does individualization matter? A randomized trial of standardized (focused) versus individualized (broad) cognitive behavior therapy for bulimia nervosa. Behaviour Research \& Therapy, 44, 273-288.

Goldstein, G. \& Hersen, M. (1998). Historical perspectives. En G. Goldstein y M. Hersen (Eds.). Handbook of Psychological Assessment. New York: Pergamon Press. (3thrd. ed.).

Hassan, I., McCabe, R. \& Priebe, S. (2007). Professional-patient communication in the treatment of mental illness: a review. Communication \& Medicine, 4, 141-152.

Hayes, S., Barnes-Holmes, D. \& Roche, B. (2001). Relational frame theory: A post-skinnerian account of human language and cognition. New York: Kluwer Academic/Plenum Publishers.

Hayes, S., \& Ju, W. (1998). The applied implications of rule-governed behavior. En W.T. O'Donohue (de), Learning and behavior therapy (pp. 374-391). Needham Heights, MA: Allyn \& Bacon.

Haynes, S. (1998). The changing nature of behavioral assessment. En A. Bellack \& M. Hersen (Eds.). Behavioral Assessment. A practical handbook. Needham Heights, MA, Allyn\&Bacon. (4 ed.).

Haynes, S. \& O’Brien, W.H. (2003). Principles and practice of behavioral assessment. NewYork: Kluwer.

Haynes, S. \& Wilson, C. (1979). Behavioral assessment: Recent advances in methods, concepts, and aplications. San Francisco, Jossey-Bass.

Hersen, M. (1988). Behavioral Assessment and psychiatric diagnosis. Behavioral Assessment, 10, 107-121.

Heyby, E. \& Haynes, S. (2003). Introduction to behavioral assessment. En E. Heiby y S. Haynes (Eds.). M. Hersen (Series Ed.).- Comprehensive handbook of psychological assessment: Vol. 3. Behavioral assessment. (pp. 3-18). New York: Wiley.

Iwata, B. A., Pace, G., Dorsey, M., Zarcone, J., Vollmer, T., \& Smith, R.. (1994). The functions of self-injurious behavior: An experimental-epidemiological analysis. Journal of Applied Behavior Analysis, 27, 215-240.

Schulte, D. (1996). Tailor-made and standardized therapy: complementary tasks in behavior therapy. A contrarian view. Journal of Behavior Therapy \& Experimental Psychiatry, 27, 119-126. 
Kanfer, F. \& Phillips, J. (1970). Learning foundations of behavior therapy. Nueva York: Wiley.

Kanfer, F. \& Saslow, G. (1965). Behavioral Analysis. Archives of General Psychiatry. 2, 529-538.

Kazdin, A. (1986). Comparative outcome studies of psychotherapy: methodological issues and strategies. Journal of Consulting and Clinical Psychology, 54, 95-105.

Leventhal, A.M. \& Rehm, L. (2005). The empirical status of melancholia: Implications for psychology. Clinical Psychology Review, 25, 25-44

Luborsky, L. \& DeRubeis, R. (1984). The use of psychotherapy treatment manuals: a small revolution in psychotherapy research style. Clinical Psychology Review, 4, 5-14.

Luciano, M.C. \& Hayes, S. (2001). Trastorno de Evitación Experiencial. International Journal of Clinical and Health Psychology, 1, 109-157.

Martell, C.R., Addis. M.E. \& Jacobson, N.S. (2001). Depression in context: strategies for guided actions. New York: Norton.

Mash, E. \& Hamsley, J. (2003). Behavioral assessment: something you get when you need. En E. Heiby \& S. Haynes (Eds.). M. Hersen (Series Ed.). Comprehensive handbook of psychological assessment: Vol. 3. Behavioral assessment. (pp. 489-502). New York: Wiley.

Matthews, G., Saklofske, D.H., Costa, P.T., Deary, I.J. \& Zeidner, M. (1998). Dimensional models of personality: a framework for systematic clinical assessment. European Journal of Psychological Assessment, 14, 36-49.

Merkelbach, H. \& Jong, P. (1997). Evolutionary models of phobias. En G. Davey (Ed.). Phobias: A Handbook of Theory, Research and Treatment (pp. 323-348). New York: Wiley.

Haynes, S. \& O’Brien, W. (1990). Functional analysis in behavior therapy. Clinical Psychology Review, 10, 649-668.

Mischel, W. \& Shoda, Y. (1995). A cognitive-affective system theory of personality: Reconceptualizing situations, dispositions, dynamics, and invariance in personality structure. Psychological Review, 102, 246-268.

Nelson, R. \& Hayes, S. (1986a). The nature of behavioral assessment. En R. Nelson y S. Hayes (Eds.). Conceptual foundations of behavioral assessment. New York: Guilford Press. 
O'Brien, W.H. \& Haynes, S. (1997). Análisis funcional de conductas. En G. Buela-Casal y J.C. Sierra (Eds.). Manual de Evaluación Psicológica. Madrid, Siglo XXI.

Pelechano, V. (1988). Del psicodiagnóstico clásico al análisis ecopsicológico. (Vol. I). Valencia: Alfaplus.

Pelechano, V. (1993). Evaluación, tests, conductismos, cognitivismos, crisis y retornos en espiral, ¿con salida?. Clínica y Salud, 4, 123-132.

Pelechano, V. (1996). Una introducción al modelo de parámetros en personalidad. En V. Pelechano (Ed.). Psicología de la Personalidad. 1. Teorías. Barcelona, Ariel.

Peñate, W. \& González, P. (1995). Estudio sobre los procedimientos diagnósticos de la práctica psicológica y psicopedagógica. Análisis y Modificación de Conducta, 21, 327-353.

Rehm, L. (1988). Assessment of depression. En A. Bellack y M. Hersen (Eds.). Behavioral Assessment. A practical handbook. New York: Pergamon Press. (3th. ed.).

Schulte, D. (1996). Tailor-made and standardized therapy: complementary tasks in behavior therapy. A contrarian view. Journal of Behavior Therapy \& Experimental Psychiatry, 27, 119-126.

Schulte, D. \& Eifert, G.H. (2002). What to do when manuals fail? The dual model of psychotherapy. Clinical Psychology: Science and Practice, 9, 312-328.

Schulte, D., Künzel, R., Pepping, G. \& Schulte-Bahrenberg, T. (1992). Tailor-made versus standardized therapy of phobic patients. Advances in Behaviour Research and Therapy, 14 , 67-92.

Sturmey, P. (2008). Behavioral Case Formulation and Intervention: $A$ Functional Analytic Approach. New York: Wiley.

Virués-Ortega, J., \& Haynes, S. N. (2005). Functional analysis in behavior therapy: Behavioral foundations and clinical application. International Journal of Clinical and Health Psychology, 5, 567-587.

Weissman, M.M., Verdeli, H., Gameroff, M.J., Bledsoe, S.E., Betts, K., Mufson, L., Fitterling, H. \& Wickramaratne, P. (2006). National survey of psychotherapy training in psychiatry, psychology, and social work. Archives of General Psychiatry, 63, 925-934.

Widiger, T.A. \& Costa, P.T. (1994). Personality and personality disorders. Journal of Abnormal Psychology, 103, 78-91. 
Wilson, G.T. (1996). Manual-based treatments: the clinical application of research findings. Behaviour Research \& Therapy, 34, 295314.

Wilson, G.T. (1997). Treatment manuals in clinical practice. Behaviour Research \& Therapy, 35,205-210.

Wilson, G.T. (2005). Psychological treatment of eating disorders. Annual Review of Clinical Psychology, 1, 439-465.

Wilson, K.G. \& Luciano, M.C. (2002). Terapia de aceptación y compromiso. Madrid: Pirámide. 
